\title{
Controlled Reversible Adsorption of Core-Shell Metallic Nanoparticles at the Polarized Water/1,2-Dichloroethane Interface Investigated by Optical Second-Harmonic Generation ${ }^{\dagger}$
}

\author{
Jean-Pierre Abid,*,» Mohamed Abid, ${ }^{\S}$ Christophe Bauer, ${ }^{\ddagger}$ Hubert H. Girault, $*$ and \\ Pierre-François Brevet ${ }^{\prime \prime}$ \\ Laboratoire d'Electrochimie Physique et Analytique, Institut des Sciences et Ingénierie Chimiques, Ecole \\ Polytechnique Fédérale de Lausanne, CH-1015 Lausanne, Switzerland, Angströmlaboratoriet, Universitet \\ Uppsala, Lägerhyddsvägen 1, SE-751 21 Uppsala, Sweden, Laboratoire de Spectrométrie Ionique et \\ Moléculaire, UMR CNRS 5579, Université Claude Bernard Lyon 1, Bâtiment Alfred Kastler, 43, \\ Boulevard du 11 Novembre 1918, F-69220 Villeurbanne cedex, France
}

Received: October 31, 2006; In Final Form: December 25, 2006

\begin{abstract}
We report the observation of the reversible adsorption of core-shell gold-silver nanoparticles at the polarized water/1,2-dichloroethane interface using the nonlinear optical technique of surface second-harmonic generation. This study unambiguously demonstrates the excellent stability against aggregation of these core-shell nanoparticles, namely, gold core nanoparticles coated with silver layers of variable thickness, in the presence of an electrolyte salt like lithium chloride. Furthermore, it is also demonstrated that the adsorption of the nanoparticles is reversible by modulating the applied potential at water/1,2-dichloroethane interface. The analysis of these results is performed within the Debye-Hückel approximation of the electrostatic interactions between the nanoparticles. This approach shows that the stability of core-shell nanoparticles can be attributed to the formation of a silver oxide layer at the surface of the particles.
\end{abstract}

\section{Introduction}

Small metallic particles (SMP) like gold and silver particles have received a large interest owing to their optical properties. These properties are dominated by the collective excitation of the free conduction band electrons of the particles known as the surface plasmon resonance (SPR). The characteristics of this resonance, namely, its energy and width, depend critically on the material, the morphology, the size, the shape, the surface charge, or the surrounding medium for instance. ${ }^{1,2}$ Tailoring these nanomaterials and elaborating assemblies therefore open new routes for the development of advanced functional materials with desired optical, magnetic, and electronic properties. During the past few years, intense efforts have been devoted to the development of new techniques of synthesis to produce welldispersed SMP with variable size or shape, new theoretical models accounting for the electronic and the optical properties of these nanoparticles, and new experimental techniques to probe isolated and assembled nanoparticles. ${ }^{3-9}$ Because of their peculiar optical and electronic properties, core-shell and homogeneous bimetallic gold-silver nanoparticles have also attracted a particular attention to understand the electronic properties of nanocomposite materials. ${ }^{10,11}$ From an optical point of view, the UV-vis photoabsorption spectra of homogeneous bimetallic gold-silver nanoparticles strongly deviate from that of core-shell gold-silver nanoparticles. In fact, a dispersed solution of bimetallic gold-silver nanoparticles exhibits a single

\footnotetext{
† Part of the special issue "Kenneth B. Eisenthal Festschrift".

* To whom correspondence should be addressed. Current address: Ecole Normale Supérieure de Cachan, Laboratoire de Physique Quantique et Moléculaire, 61, avenue du Président Wilson, 94235 Cachan cedex, France. E-mail: jpabid@lpqm.ens-cachan.fr.

Ecole Polytechnique Fédérale de Lausanne.

$\S$ Universitet Uppsala.

" Université Claude Bernard Lyon 1.
}

plasmon resonance located at an energy between the SPR of pure silver nanoparticles at $3.1 \mathrm{eV}$ and pure gold nanoparticles at $2.38 \mathrm{eV}$ whereas a dispersed solution of core-shell goldsilver nanoparticles exhibits two SPR located at 3.1 and 2.38 $\mathrm{eV}$. This experimental feature is now well understood in terms of Mie theory extended to core-shell nanoparticles. ${ }^{1,12-14}$ Although an increasing amount of work is being devoted to the linear optical properties of nanoparticles, little attention has been given to their nonlinear optical properties. ${ }^{15-18}$ Even scarcer is the work reported of the nonlinear optical properties of core-shell nanoparticles. ${ }^{19}$ Nonlinear optical techniques like surface second-harmonic generation (SSHG) and sum frequency generation (SFG) have gained popularity, however, as surface probe to investigate systems like liquid/metal, air/liquid, and liquid/liquid interfaces. ${ }^{20-27}$ This interest lies in the property of even-order nonlinear optical processes which vanish in the dipole approximation in media with inversion symmetry. As a result, at interfaces between two centrosymmetric media like liquids, the SHG process is allowed and highly surface specific.

In this work, we report the use of SHG to investigate the adsorption of gold nanoparticles coated with variable thicknesses of silver at the polarized water/1,2-dichloroethane interface. Stock solutions of particles were prepared chemically prior to their intoduction into the water phase. The potential control between the two adjacent phases was externally controlled and allowed for the observation of the controlled reversible adsorption of the core-shell nanoparticles. No aggregation was observed for these composite core-shell particles and this feature may be understood within a Debye-Huckel model of the electrostatic interactions.

\section{Experimental}

Core-Shell Nanoparticles Synthesis. Tetrachloroauric acid $\left(\mathrm{HAuCl}_{4}, 3 \mathrm{H}_{2} \mathrm{O}, 99.9999 \%\right)$ and silver nitrate $\left(\mathrm{AgNO}_{3}, 99.9999 \%\right)$ 


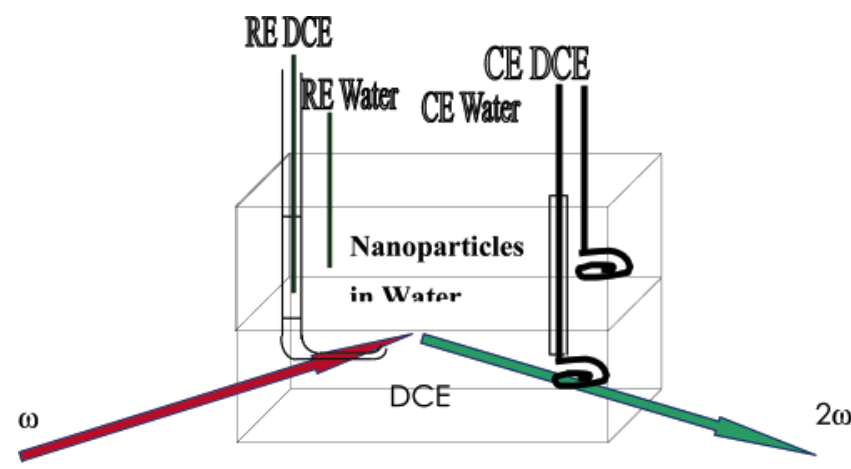

Figure 1. Schematics of the electrochemical cell employed in the SHG experiments. (RE DCE, reference electrode in DCE; CE DCE, counter electrode in DCE; RE Water, reference electrode in water; CE Water, counter electrode in water.)

were purchased from Aldrich. Citrate trisodium dihydrate $\left(\mathrm{Na}_{3} \mathrm{C}_{6} \mathrm{H}_{5} \mathrm{O}_{7}, 2 \mathrm{H}_{2} \mathrm{O}\right)$ and hydrochloric and nitric acids were purchased from Fluka. All chemicals were used as received. Prior to use, the glassware and the magnetic stirring beads were thoroughly cleaned with freshly prepared aqua regia followed by extensive rinsing with Millipore water (conductivity $0.8 \mu \mathrm{S}$ $\left.\mathrm{cm}^{-1}\right)$.

Silver-coated gold nanoparticles were prepared with a variable amount of silver in a two-step procedure as reported previously. ${ }^{19}$ Core-shell nanoparticles with a molar ratio $x$ ranging from 0 to 0.5 were synthesized and studied at water/1,2dichloroethane. The solutions were used as prepared without further treatments.

Electrochemical Cell. Lithium chloride, bis(triphenylphosphorandylidene) ammonium chloride (BTPPACl), tetrabutylammonium chloride (TBACl), tetramethylammonium chloride (TMACl), and 1,2-dichloroethane (DCE) were all purchased from Fluka. Potassium tetrakis(4-chlorophenyl)borate (KTPBCl) was purchased from Biosolved. The organic supporting salt (BTPPATPBCl) was prepared by metathesis of bis(triphenylphosphoranylidene ammonium chloride and potassium tetrakis (4-chlorophenyl)borate in a 3:1 mixture of methanol and water and then was recrystallized twice in acetone. A system of four electrodes was designed to apply and control the potential between the two immiscible liquids. The two counter electrodes were made of platinum and the reference electrodes were made of $\mathrm{AgCl}$ coated silver wires. The SHG experiments were performed in a rectangular quartz cell obtained from Hellma. The reference to the potential scale of the Galavani potential difference $\Delta_{0}{ }^{\mathrm{w}} \phi$ was defined using the standard ion transfer potential of $\mathrm{TMA}^{+}$, the value of which is $+0.160 \mathrm{~V}$. Figure 1 gives a schematic of the experimental cell.

Nanoparticle Characterization. The UV-vis photoabsorption spectra of the different solution of silver-layered gold particles were performed with a spectrophotometer (USB 2100, Ocean Optics, United States) in the range 190-800 nm. The final spectra were obtained by averaging over 600 spectra. The size distribution of the pure gold and gold-silver core-shell nanoparticles was determined by transmission electron microscopy (TEM) (CM20, Philips) and dynamic light scattering. High-resolution transmission microscopy (HRTEM) (CM300, Philips) was also performed to yield the thickness of the silver layer. For all TEM measurements, a drop of the sample solution was deposited on a copper grid and was dried under nitrogen atmosphere in a glove box. The size distribution was obtained from a collection of 300 particles belonging to 10 different batches.
Nonlinear Optical Experiments. The rectangular quartz cell $(4 \mathrm{~cm} \times 2 \mathrm{~cm})$ was first filled with $12 \mathrm{~mL}$ of a $2.5 \mathrm{mM}$ BTPPATPBCl 1,2-dichloroethane solution. On top of this organic phase, a volume of $8 \mathrm{~mL}$ of a $2.5-\mathrm{mL} \mathrm{LiCl}$ aqueous solution was introduced. The core-shell nanoparticles were introduced directly into this phase by direct addition of adequate aliquots. The SHG experiments were performed with the idler output of a Q-Switched Nd:YAG laser pumped optical parametric oscillator (OPO) delivering pulses of $20 \mathrm{~mJ}$ and 5-ns duration at a wavelength of $1064 \mathrm{~nm}$ and a repetition rate of $10 \mathrm{~Hz}$. The output signal from the OPO can be tuned from 770 to $1300 \mathrm{~nm}$ with an energy varying from 20 to $1.5 \mathrm{~mJ}$ depending on the wavelength. The incident beam was collimated by a telescope to a diameter of $3.5 \mathrm{~mm}$ and was directed with a periscope onto the water/DCE interface through the organic phase. The experimental angle between the incident beam and the surface normal was about $69.5^{\circ}$ and ensured that the experiment was performed in a total internal reflection optical geometry (TIR). A set of colored filters were used just before the quartz cell to reject any second-harmonic light generated from the optics placed before the cell. The reflected SHG signal was then passed through a colored filter to reject light at the fundamental wavelength and was focused with a lens onto the entrance slit of a monochromator coupled to a fast response photomultiplier tube (Hamamatsu, R928), the output signal of which was sent to a gated boxcar averager. The fundamental input and output polarization angles were controlled by a halfwave plate on the entrance side and a UV-analyzer on the ouput side of the setup. In all the experiments performed on the coreshell nanoparticles at water/DCE and reported here, the fundamental and the harmonic light beams polarization state were set parallel to the plane of incidence (linear p polarization state).

\section{Results and Discussion}

UV Photoabsorption Spectroscopy and TEM Imaging. Figure 2a gives the UV-vis photoabsorption spectra of the gold nanoparticle solution before and after silver coating. The SPR for the aqueous pure gold nanoparticle solution occurs at 2.38 $\mathrm{eV}$, a wavelength of about $520 \mathrm{~nm}$. The regular increase of the absorbance at energies above $2.58 \mathrm{eV}$, or wavelengths lower than $480 \mathrm{~nm}$, arises from the interband transitions. In the case of the core-shell nanoparticles, a large increase of the absorbance is observed between 2.4 and $5 \mathrm{eV}$ with the increasing amount of silver deposited onto the surface of the gold nanoparticles. The absorption spectra therefore present two broad resonances located at 3.1 and $2.4 \mathrm{eV}$ for these heterogeneous particles. Because of the existence of two distinct metallic phases, two SPR are observed at the energy location corresponding to the location of the corresponding pure metal particles. The low-energy resonance corresponding to the gold SPR gradually shifts toward higher energies, eventually being hidden by the large higher energy SPR of silver. Simultaneously, the silver SPR increases and shifts to lower energies progressively. The absence of a single SPR at an intermediate energy position between the two SPR at 3.1 and $2.4 \mathrm{eV}$ is a clear indication of the absence of isolated alloyed gold-silver nanoparticles in the solution that could be formed during the silver reduction and deposition process. Alloying between silver and gold could indeed occur by atomic interdiffusion because of the similar atomic properties of these metals. ${ }^{28}$ Theoretical approaches based on the Mie theory and its developments for core-shell structures yield a good agreement further supporting this analysis. ${ }^{12-14}$ For a dilute suspension of small metallic 


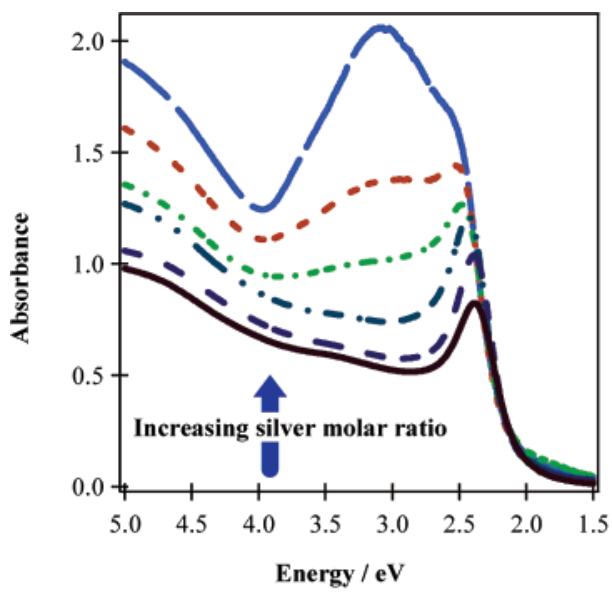

(a)

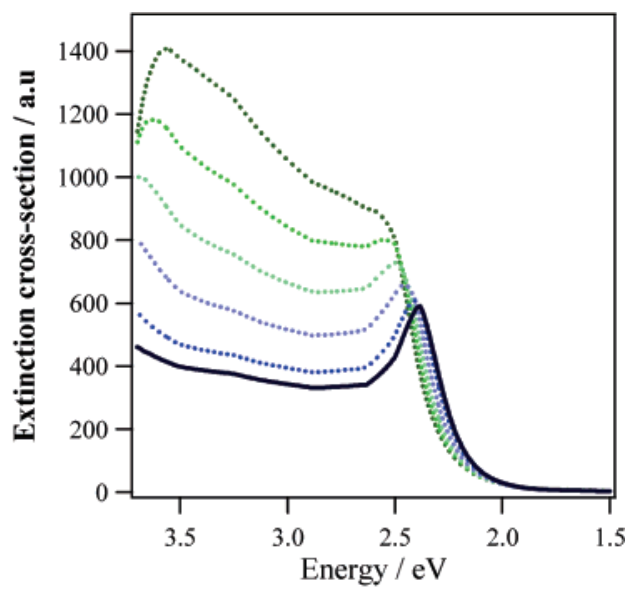

(b)

Figure 2. (a) UV-vis absorption spectra recorded for gold core-silver shell particles with a silver molar fraction ranging from 0 to 0.5 . (Bottom solid curve: seeding gold particles, dashed line curves: increasing amount of silver from 0.1 to 0.5 ). (b) Theoretical extinction cross section for core-shell nanoparticles in the UV-vis range (from bottom to top: core-shell nanoparticles with silver molar fraction ranging from 0 to 0.5 ).

TABLE 1: Size Characteristics of Gold and Core-Shell Nanoparticles

\begin{tabular}{ccc}
\hline silver fraction & average size/nm & layer thickness/nm \\
\hline $\mathbf{0}$ & 16.0 & 0 \\
$\mathbf{0 . 1}$ & 17.1 & 0.5 \\
$\mathbf{0 . 2}$ & 17.9 & 1.0 \\
$\mathbf{0 . 3}$ & 18.7 & 1.3 \\
$\mathbf{0 . 4}$ & 19.8 & 1.9
\end{tabular}

particles without any interaction between particles, the extinction cross section for a particle if radius $R$ is given by ${ }^{13}$

$$
\begin{aligned}
& C_{\text {ext }}=\frac{4 \pi R^{2} \epsilon_{\mathrm{m}}{ }^{1 / 2}}{\lambda} \\
& {\left[\frac{\left(\epsilon_{\text {shell }}-\epsilon_{\mathrm{m}}\right)\left(\epsilon_{\text {core }}-\epsilon_{\mathrm{m}}\right)+(1-f)\left(\epsilon_{\text {core }}-\epsilon_{\text {shell }}\right)\left(\epsilon_{\mathrm{m}}+2 \epsilon_{\text {shell }}\right)}{\left(\epsilon_{\text {shell }}+2 \epsilon_{\mathrm{m}}\right)\left(\epsilon_{\text {core }}+2 \epsilon_{\text {shell }}\right)+(1-f)\left(2 \epsilon_{\text {shell }}-2 \epsilon_{\mathrm{m}}\right)\left(\epsilon_{\text {core }}-\epsilon_{\text {shell }}\right)}\right]}
\end{aligned}
$$

where $\epsilon_{\text {core }}, \epsilon_{\text {shell }}$, and $\epsilon_{\mathrm{m}}$ are, respectively, the dielectric functions of the metal core, metal shell, and surrounding medium and $f$ is the volume fraction of silver layer. The resulting absorption spectra calculated for different silver layer thicknesses are shown in Figure $2 b$ using the dielectric functions of the corresponding bulk metals. ${ }^{29}$ No deposition or aggregation was observed for these as-prepared sols and the solutions were stable for more than 6 months. As described elsewhere, ${ }^{19}$ the gold nanoparticles presented a near-spherical shape with an average size of $16 \mathrm{~nm}$ as deduced from a statistical analysis of the TEM imaging and further complemented by dynamic light-scattering measurements. The solution was monodispersed with a standard deviation of about $12 \%$. With the growth of a silver layer shell, the average diameter of the particles increased from $16 \mathrm{~nm}$ up to $19.8 \mathrm{~nm}$, see Table 1 . High-resolution TEM measurements were also performed on the silver shell-gold core particles, see Figure 3. If the thin layer obtained for the $\mathrm{Au}_{0.9} \mathrm{Ag}_{0.1}$ is barely seen, the layer obtained for the $\mathrm{Au}_{0.6} \mathrm{Ag}_{0.4}$ is clearly observed with a slight variation of the thickness depending on the gold crystal plane where the growth occurred.

Stability of Charged Nanoparticles. The stabilization of the SMP is achieved electrostatically with the adsorption of ions such as chloride or citrate. These adsorbed ions provide the electrostatic repulsion required to avoid the process of aggregation between the nanoparticles. As a result, the double layer formed at the interface between the particle and the solution induces a modification of the dielectric properties of the particles. However, the consequences on the energy location of the SPR were not seen here, possibly hindered by other phenomena like the effect of the surrounding solvent and its correct description. ${ }^{30}$ In principle, any weakening of the Coulombic interactions as compared to the van der Waals attraction will cause the aggregation of the metal particles and hence the modification of their optical properties through electromagnetic coupling. In a suspension of aggregates, the optical reponse strongly deviates from the model developped for well-dispersed nanoparticles in solution. Different models may be derived depending on the state of the aggregation, but this suggests that the stability of metallic particle suspension can be monitored through its optical spectrum.

In small metal particles, surface-bound ions determine the electrostatic interactions between neighboring particles. Aggregation is thus initiated by changes in the double layer like the addition of a salt, for instance, sodium chloride or sulfate, to screen the particle surface charge. Depending on the screening efficiency, ${ }^{31}$ the van der Waals attractive contribution will be overcome by the electrostatic repulsive part, leading to the aggregation process. In electrochemistry, salts like lithium chloride or sulfate are often used as supporting electrolytes. In the perspective of the nonlinear optical experiments performed in an electrochemical context, the effect of lithium chloride on the stability of the gold and the gold-silver core-shell nanoparticles was studied. First, variable amounts of lithium chloride were added in the particle suspensions. Second, a fixed concentration of $2.5 \mathrm{mM}$ lithium chloride in an aqueous phase was used, the latter corresponding to the salt concentration used in a typical electrochemical cell at water/DCE for the aqueous supporting electrolyte. For the suspension of pure gold nanoparticles, the evolution of the UV-vis absorbance with the lithium chloride concentration is presented in Figure 4a. Initially, with a salt concentration of $8 \mathrm{mM}$, the absorbance is decreased and a new band appears at photon energies below $2 \mathrm{eV}$. At salt concentrations exceeding $16.6 \mathrm{mM}$, the UV-vis absorption spectrum is, however, dramatically modified with the almost complete disappearance of the SPR and the simultaneous rise of the new absorption band at energies below $2 \mathrm{eV}$. This new low-energy band can be associated with the aggregation process and the interparticle interactions. The latter process though has a slow kinetics and after 10 min only is complete. No further evolution of the spectrum is observed afterward at a higher salt 


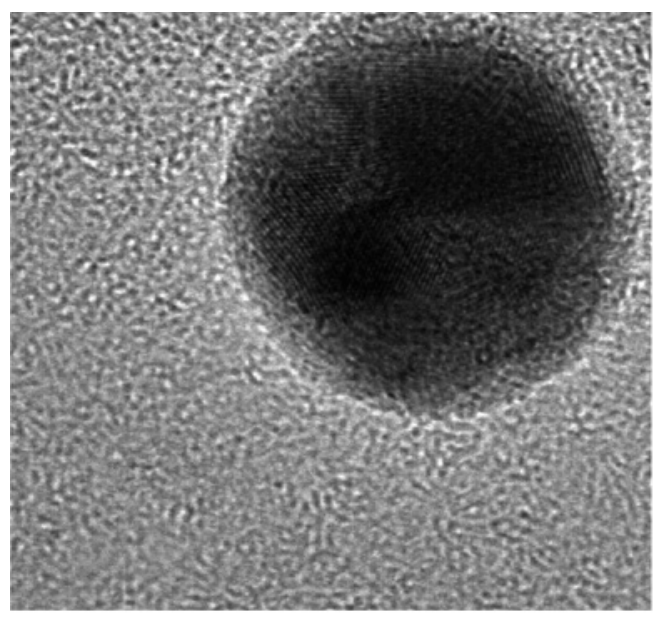

(a)

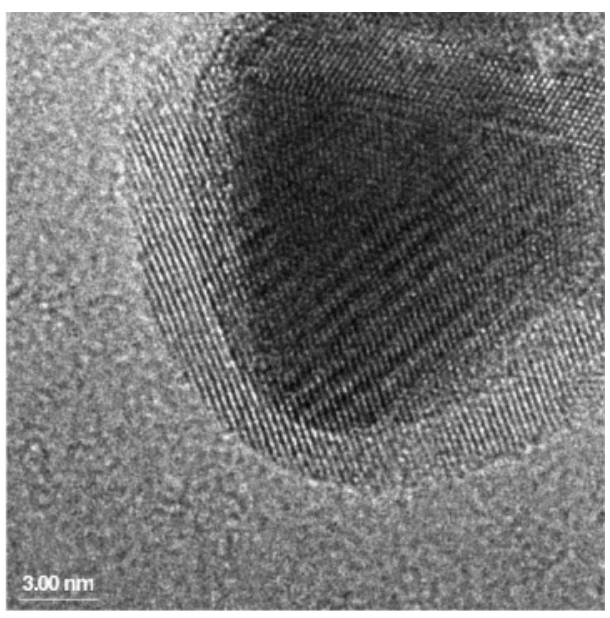

(b)

Figure 3. (a) HRTEM image of a core-shell particle with a silver molar fraction of 0.1. (b) HRTEM image of a core-shell particle with a silver molar fraction of 0.4 .

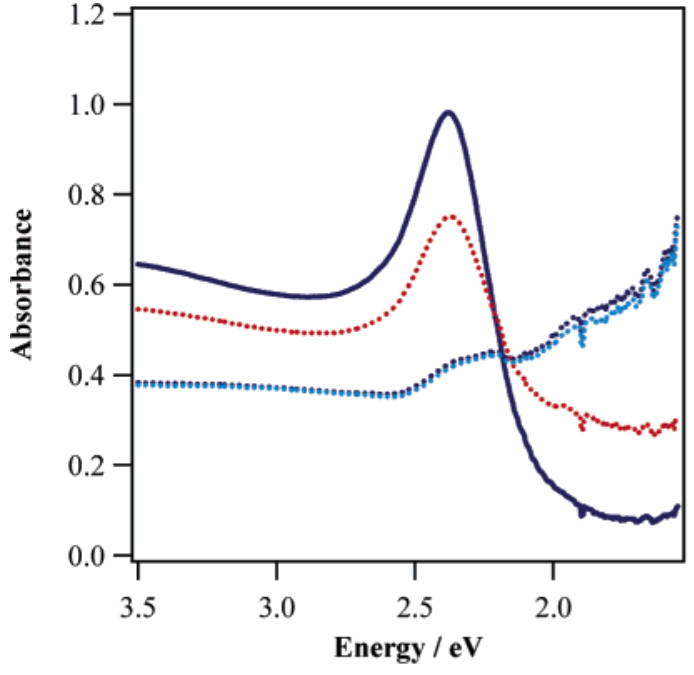

(a)

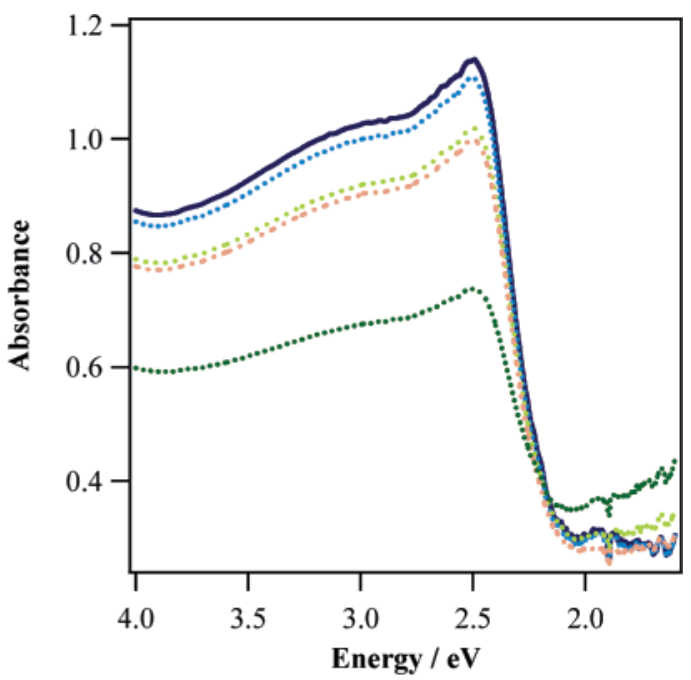

(b)

Figure 4. (a) Effect of the lithium chloride salt additions on the stability of the gold particle suspension as followed by UV-vis absorption spectroscopy: solid line, gold particles without lithium chloride; dotted line, from top to bottom: 8, 17, and $32 \mathrm{mM}$ lithium chloride concentration. (b) Effect of the lithium chloride salt additions on the stability of $\mathrm{Au}_{0.6} \mathrm{Ag}_{0.4}$ particle suspension as followed by UV-vis absorption spectroscopy: solid line, $\mathrm{Au}_{0.6} \mathrm{Ag}_{0.4}$ particles without lithium chloride; dotted line, from top to bottom: 8, 16, 32, and $66 \mathrm{mM}$ lithium chloride concentration.

concentration. Within the same range of the lithium chloride concentration, no dramatic evolution of the absorption spectrum of the gold core-silver shell particle suspension is observed, see Figure 4b. In this set of experiments, a suspension of $\mathrm{Au}_{0.6^{-}}$ $\mathrm{Ag}_{0.4}$ was used. A salt concentration of $60 \mathrm{mM}$ is required to induce the start of the aggregation of the nanoparticles as indicated by the start of the rise of the absorbance at photon energies below $2 \mathrm{eV}$. Hence, compared to the gold nanoparticles suspension, a better stability is observed for the core-shell nanoparticles as compared to the pure gold ones. Considering the mechanism of aggregation, this feature can be attributed to a higher surface charge on the core-shell particles. Similarly, the presence of a silver oxide layer reducing the van der Waals interactions could play a nonnegligible role. These investigations were repeated at the water/DCE interface with the nanoparticles suspension used as the upper aqueous phase of the liquid/liquid cell. This time, the salt concentration was held constant at 2.5 $\mathrm{mM}$, the salt concentration used in the nonlinear optical experiments coupled to the electrochemical measurements. The UV-vis absorption measurements were performed with two optical fibers connected to the spectrophotometer and employed in a transmission mode right through the whole two-phase system. The results obtained are displayed in Figure 5a and 5b. In the case of the gold nanoparticle suspension, the aggregation process does occur albeit with a slower kinetics. Ninety minutes after the start of the experiment, the aggregation process is clearly visible. This time, the absorption spectrum is modified both at higher and lower photon energies compared to the SPR. This feature arises from the measurement procedure. In the previous geometry, large aggregates will sediment and move downward away from the light path in the photoabsorption cell. On the opposite, in the second configuration, the large aggregates will sediment at the interface and therefore will remain within the light path in the cell. The increase of the absorbance at photon energies higher than the SPR one should be attributed thus to the large aggregates. At photon energies around $2 \mathrm{eV}$, the increase of the absorbance is similar in the two configurations. The core-shell nanoparticle suspension presents a rather different behavior. After $2000 \mathrm{~min}$, no evidence for any aggregation is observed, in particular at low photon energies 


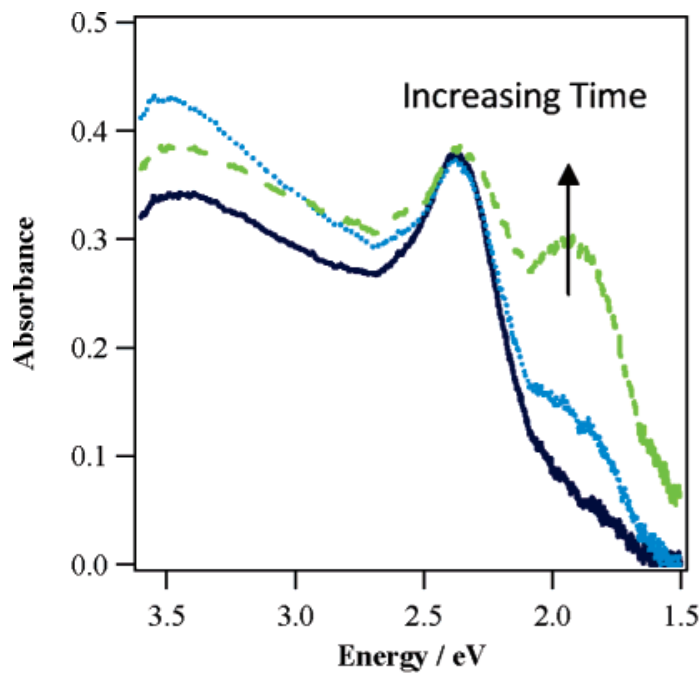

(a)

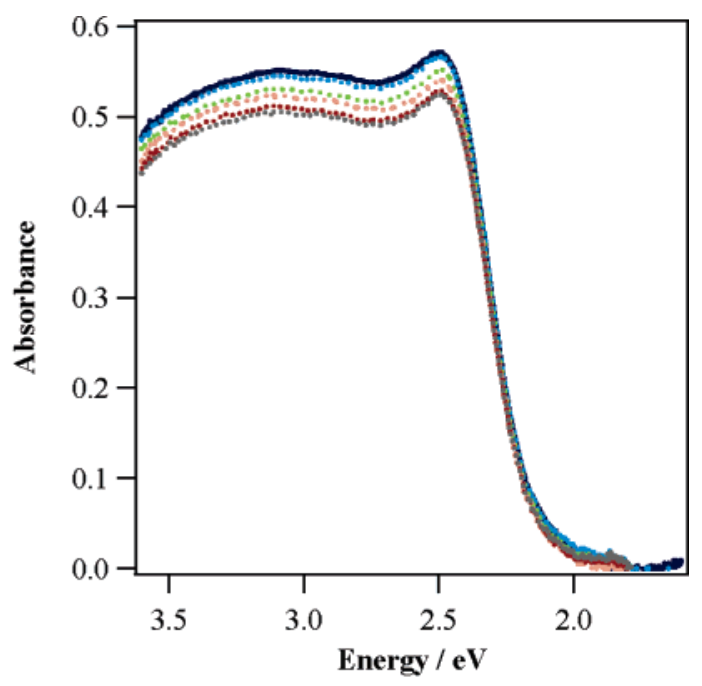

(b)

Figure 5. (a) Time evolution of the UV-vis absorption spectrum of gold nanoparticles at the water/DCE interface with a lithium chloride concentration of $2.5 \mathrm{mM}$ : solid line, $t=0$; dotted line, $t=90 \mathrm{~min}$; dashed line, $t=720 \mathrm{~min}$. (b) Time evolution of the UV-vis absorption spectrum of $\mathrm{Au}_{0.6} \mathrm{Ag}_{0.4}$ particles at the water/DCE interface with a lithium chloride concentration of $2.5 \mathrm{mM}$ : solid line, $t=0$; dotted line, from top to bottom: $t=90,720,1000,1200,2000 \mathrm{~min}$.

around $2 \mathrm{eV}$. In the range of energies above $2.5 \mathrm{eV}$, a small progressive decrease of the absorbance is observed. This feature is difficult to describe. It is possible that long-range interactions do occur between particles, enough to decrease the absorbance in this range of energies in a way similar to the homogeneousphase aggregation process but not sufficiently to produce the rise of a new band at low photon energies. In the liquid-liquid configuration, additional complications must also be taken into account like the effect of the interface or the solubility of the particles in both phases, and these could play a nonnegligible role. Nevertheless, these results obtained in two different geometries for two different particle morphologies can be understood in term of the changes of the surface charge retained by the particles or the formation of a silver oxide layer at the surface of the core-shell particles. In fact, the stability of dispersed nanoparticles is governed by the surface charge and on the extent of the electrical double layer.

The repulsive electrostatic pair potential between two charged spheres large enough that the Debye-Hückel approximation holds is given by the Derjaguin-Landau-Verwey-Overbeek (DLVO) theory and writes ${ }^{32}$

$$
V_{\mathrm{DLVO}}(x)=\frac{Z^{2} e^{2}}{\epsilon_{0} \epsilon_{\mathrm{m}}} \frac{e^{-\kappa x}}{(1+\kappa R) x}
$$

where $x$ is the distance separation between the particles, $Z$ is the particle surface charge, $R$ is the radius of the particle, and $\kappa$ is the inverse screening length. Also, $\epsilon_{0}$ is the permittivity of the free space and $e$ is the charge of the electron. This electrostatic repulsive potential is compensated by an attraction potential because of the van der Waals interactions. The expression of this attractive potential is given by

$$
\begin{array}{r}
V_{\mathrm{vdW}}(x)=-A_{\mathrm{H}}\left[\frac{2 R^{2}}{\left(x^{2}+4 R x\right)}+\frac{2 R^{2}}{x^{2}+4 R x+4 R^{2}}+\right. \\
\left.\ln \left(\frac{x^{2}+4 R x}{x^{2}+4 R x+4 R^{2}}\right)\right]
\end{array}
$$

where $A_{\mathrm{H}}$ is the Hamaker constant. The stability of the dispersed suspension of nanoparticles will then depend on the total interaction energy, namely,

$$
V_{\text {Total }}(x)=V_{\mathrm{DLVO}}(x)+V_{\mathrm{vdW}}(x)
$$

To understand the discrepancy between the different stability of the gold and the gold core-silver shell nanoparticles, the total interaction energy has been estimated using the effective charges of the particles determined using capillary electrophoresis. The Hamaker constants for the nanoparticles have been taken from ref 33. In the same way, the estimated interparticle potential functions are represented in Figure $6 \mathrm{a}$ and $6 \mathrm{~b}$ expressed in units of $k_{\mathrm{B}} T$. For both the gold and the core-shell nanoparticles, before the addition of the salt, the screening length is estimated to be equal to $3.5 \mathrm{~nm}$. Hence, without addition of the lithium chloride salt, the repulsive interaction easily overcomes the attractive one and no aggregation phenomenon occurs. However, as observed in Figure 6a, addition of lithium chloride severely reduces the height of the repulsive barrier. In the case of the gold nanoparticles, the low surface charge cannot provide a sufficient repulsive interaction and the aggregation process cannot be avoided. On the opposite, for the core-shell particles, the much stronger surface charge prevents the aggregation. The deposition of the silver layer around the gold nanoparticles increases the particle surface effective charge by a factor of nearly 4 (62 for gold nanoparticles and 235 for $\mathrm{Ag}_{0.4} \mathrm{Au}_{0.6}$ ), providing a better stabilization of the core-shell particles as compared with the gold ones. This increase in surface charge cannot be related to the much smaller increase in surface area because of the larger radius of the particle. It has therefore to be related to a higher surface density of citrate and chloride anions. For the core-shell particles, the enhanced effective charge allows the stability of the suspension with a final screening length of about $1.5 \mathrm{~nm}$. Finally, a silver oxide layer may be formed at the surface of the core-shell particles. This existence of this layer would change both the attractive and the repulsive potentials together.

In the liquid/liquid configuration, the process of aggregation is observed for the gold nanoparticles at concentrations of the lithium chloride salt well below the concentrations required to 


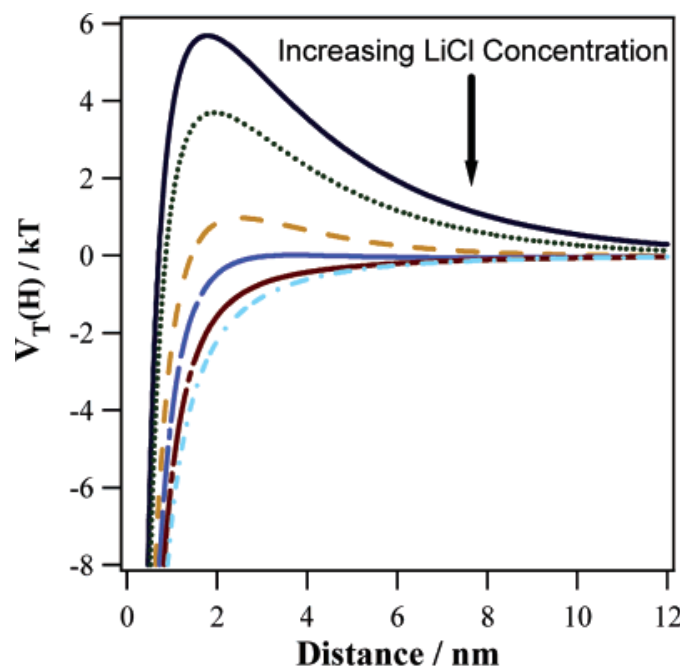

(a)

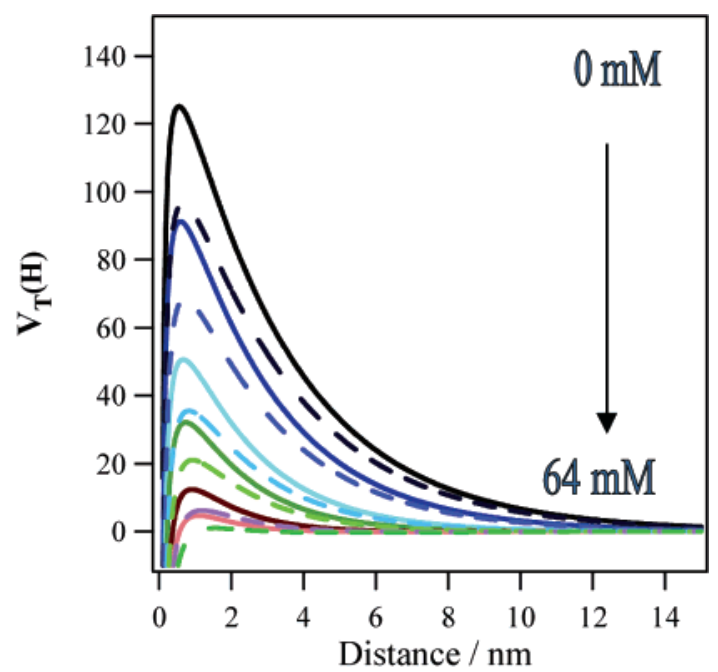

(b)

Figure 6. (a) Theoretical curves of the total potential of interaction between two particles in the presence of lithium chloride salt with different concentrations for gold particles: From top to bottom: 0, 2.5, 8.3, 16.6, 32.3, $64 \mathrm{mM}$. (b) Theoretical curves of the total potential of interaction between two particles in the presence of lithium chloride salt with different concentrations for core-shell particles: From top to bottom: 0 , 2.5, 8.3, 16.6, 32.3, $64 \mathrm{mM}$, (solid lines) $\mathrm{Au}_{0.9} \mathrm{Ag}_{0.1}$ core-shell particles, (dashed lines) $\mathrm{Au}_{0.6} \mathrm{Ag}_{0.4}$ core-shell particles.

induce the aggregation process in the homogeneous phase. This feature may be related to the difference in solubility of the nanoparticles in the two phases and also the different dielectric properties of the aqueous and the organic phase. Furthermore, the Hamaker constant is also dependent on the solvent where the particles are dispersed. The strong difference in stability between the two type of particles, and more particularly the absence of aggregation observed for the gold core-silver shell particles, suggests that it is possible to conduct optical experiments at the polarized liquid-liquid interface without the complication of aggregation.

SHG Studies at the Polarized Water/DCE Interface. The study of the adsorption of the nanoparticles was then undertaken at the polarized water/DCE interface. The Galvani potential between the two phases was swept forward and backward from 0.55 to $-0.20 \mathrm{~V}$ and the SHG experiments where conducted at a harmonic wavelength in resonance with the SPR of the dispersed nanoparticles. In this case, the SHG signal intensity is enhanced. ${ }^{16}$ Previous works by Agarwal and Jha have demonstrated in the past that this enhancement should occur when $\{\operatorname{Re}\} \epsilon(\omega)+2 \epsilon_{\mathrm{m}}=0 .{ }^{34}$ The $\mathrm{SH}$ intensities were thus measured at $2.39 \mathrm{eV}$ (harmonic wavelength of $518 \mathrm{~nm}$ ) and $2.53 \mathrm{eV}$ (harmonic wavelength of $490 \mathrm{~nm}$ ). The evolution of the $\mathrm{SH}$ intensity with respect to the Galvani potential of the water/DCE interface is displayed in Figure 7. As Galvani potential is swept toward negative, the SH signal starts to appear and reaches its maximum at the most negative potentials. This feature has been observed for core-shell nanoparticles with different silver mole fractions. On the opposite, if the Galvani potential is swept positive, the SH intensity decreases back to its background level close to zero. However, if the Galvani potential is repeatedly swept back and forth negative to positive, the first initial cycle for the $\mathrm{SH}$ intensity is repeated without any loss in intensity. The SHG clearly shows a reversible nature and an intensity controlled by the externally applied potential. For the core-shell nanoparticles, no $\mathrm{SH}$ signal is observed at energies lower than $2.3 \mathrm{eV}$ corresponding to the energy of the SPR of pure gold particles. These low energies correspond to the range of energies where a new absorption band was observed during the aggregation process in the homogeneous phase. It is therefore concluded that aggregation is not occurring at the

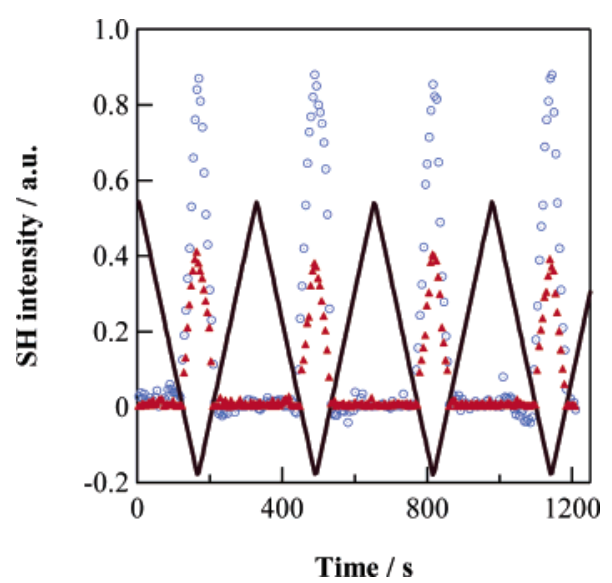

Figure 7. SHG intensity and Galvani potential difference as a function of time at the water/DCE interface for (triangles) $\mathrm{Au}_{0.9} \mathrm{Ag}_{0.1}$ particles and (circles) $\mathrm{Au}_{0.6} \mathrm{Ag}_{0.4}$ particles.

polarized water/DCE interface and that the adsorption process is indeed reversible. On the opposite, in the case of the gold nanoparticles, an initial sweep of the galvanic potential toward negative yields an increase of the SHG intensity followed by a decrease when the potential is swept positive. However, no further cycle may be performed since the SHG signal vanishes. This vanishing of the SHG signal is attributed to the aggregation process and the loss of the SPR enhancement. These results clearly demonstrate that the better stability of the core-shell nanoparticles allows the control through the applied potential of the reversible adsorption of these nanomaterials owing to the high surface charge held by the composite particles. At negative Galvani potential differences, the particles are accumulated at the interface yielding an increase of the SHG intensity, whereas for positive Galvani potential differences, desorption of the particles yields the decrease of the SHG signal intensity. This behavior suggests that the increase and decrease of the SHG intensity is determined by the density of the particles at the interface since the SHG intensity writes ${ }^{19-23}$

$$
I_{\mathrm{SHG}}=K\left|\chi_{S}^{(2)}\right|^{2} I^{2}
$$

where $I$ is the fundamental intensity and $K$ is a constant 
depending on the geometry of the experiment and the dielectric constants of the two liquid phases. The susceptibility tensor $\chi_{S^{(2)}}$ of the interface also writes

$$
\chi_{\mathrm{S}}^{(2)}=N_{\mathrm{S}}\langle\beta\rangle
$$

where $N_{\mathrm{s}}$ is the surface density of the particles and $\langle\beta\rangle$ is the hyperpolarizability of the nanoparticles. The averaging procedure over all orientation may be required since the particles may not be purely spherical and may have a pure dipolar response. ${ }^{18}$ In absence of aggregation, the hyperpolarizability may be assumed constant and therefore the SHG intensity should scale as the square of the particle surface density. The observed experimental SHG intensity is, though, not straightforward to extract and relate to the particle surface density since the potential dependence of the adsorption isotherm is not precisely known. ${ }^{35}$ In the case of the gold nanoparticles, a similar behavior is observed during the first cycle with the accumulation of the particles at the interface. Because of the weakness of the effective surface charge of these particles, aggregation is initiated, however, inducing a red shift of the SPR. The SHG intensity thus subsequently vanishes by loss of the resonance enhancement and no further cycle may be observed.

\section{Conclusions}

Gold core-silver shell nanoparticles were chemically prepared using a seeding procedure starting from pure gold particles and were electrostatically stabilized. These nanoparticles present an enhanced stability in aqueous solutions and at the water/ DCE interface as compared to the pure gold particles because of a nearly 4-fold increase in surface charge and the possible presence of silver oxide at the surface. SHG experiments were performed demonstrating the reversible adsorption and desorption at the interface controlled by the externally applied potential without any sign of aggregation. For gold nanoparticles, the weakness of the surface charge quickly induced the aggregation of the nanoparticles at liquid/liquid interface and the loss of the SHG intensity precluding the observation of any reversibility.

\section{References and Notes}

(1) Kreibig, U.; Vollmer, M. In Optical Properties of Metal Clusters; Springer Series in Materials Science, Volume 25; Berlin, 1997.
(2) Kelly, K. L.; Coronado, E.; Zhao, L. L.; Schatz, G. C. J. Phys. Chem. B 2003, 107, 668 .

(3) Daniel, M. C.; Astruc, D. Chem. Rev. 2004, 104, 293.

(4) Mirkin, C. A.; Letsinger, R. L.; Mucic, R. C.; Storhoff, J. J. Nat. 1997, 382, 607.

(5) Mafune, F.; Kohno, J. Y.; Takeda, Y.; Kondow, T. J. Phys. Chem. B 2002, 106, 7575 .

(6) Wang, Z.; Tan, B.; Hussain, I.; Schaeffer, N.; Brust, M.; Cooper, A. I. Langmuir 2006, ASAP.

(7) Watabe, M.; Furukawa, S.; Akamashi, S; Oda, T. Bio Industry 2005, 22,60 .

(8) Siegel, R. W. J. Mater. Res. 1998, 3, 1367.

(9) Arbouet, A.; Christophilos, D.; Del fatti, N.; Vallée, F.; Huntzinger, J. R.; Arnaud, L.; Billaud, P.; Broyer, M. Phys. Rev. Lett. 2004, 93, 127401. (10) Mulvaney, P. Langmuir 1996, 12, 788.

(11) Hodak, J. H.; Henglein, A.; Giersig, M.; Hartland, G. V. J. Phys. Chem. B 2000, 104, 11708 .

(12) Mie, G. Ann. Phys. 1908, 25, 377.

(13) Bohren, C. F.; Huffman, D. R. Absorption and Scattering of Light by Small Particles; Wiley-Interscience: New York, 1983.

(14) Aden, A. L.; Kerker, M. J. Appl. Phys. 1951, 22, 2242.

(15) Hache, F.; Ricard, D.; Flytzanis, C. J. Opt. Soc. Am. B 1986, 3 , 1646 .

(16) Antoine, R.; Brevet, P. F.; Girault, H. H.; Bethell, D.; Schiffrin, D. J. Chem. Commun. 1997, 1901

(17) Vance, F. W.; Lemon, B. I.; Hupp, J. T. J. Phys. Chem. B 1998, 102, 10091 .

(18) Nappa, J.; Revillod, G.; Russier-Antoine, I.; Jonin, C.; Benichou, E.; Brevet, P. F. Phys. Rev. B 2005, 71, 165407.

(19) Abid, J-P.; Nappa, J.; Girault, H. H.; Brevet, P. F. J. Chem. Phys. 2004, 121, 12577 .

(20) Shen, Y. R. In Chemistry and Structure at Interfaces. New laser and optical techniques; Hall, R. B., Ellis, A. B., Eds.; VCH Publishers: New York, 1986.

(21) Eisenthal, K. B. Ann. Rev. Phys. Chem. 1992, 43, 627.

(22) Corn, R. M.; Higgins, D. A. Chem. Rev. 1994, 94, 107.

(23) Brevet, P. F.; Girault, H. H. In Liquid-Liquid Interfaces : Theory and Methods; Volkov, A. G., Dreamer, D. W., Eds.; CRC Press: Boca Raton, FL, 1996

(24) Eisenthal, K B. Chem. Rev. 1996, 96, 1343.

(25) Ong, S.; Zhao, X.; Eisenthal, K. B. Chem. Phys. Lett. 1992, 191, 327.

(26) Castro, A.; Sitzmann, E. V.; Zhang, D.; Eisenthal, K. B. J. Phys. Chem. 1991, 95, 6752.

(27) Sitzmann, E. V.; Eisenthal, K. B. J. Phys. Chem. 1988, 92, 4579

(28) Moskovits, M.; Srnova-Sloufova, I.; Vlckova, B. J. Chem. Phys. 2002, 116, 10495

(29) Johnson, P. B.; Christy, R. W. Phys. Rev. B 1972, 8, 4370.

(30) Bohren, C. F.; Hunt, A. J. Can. J. Phys. 1977, 55, 1930.

(31) Hunter, R. J. Foundations of Colloid Science; Oxford University Press: Oxford, U.K., 2001.

(32) Crocker, J. C.; Grier, D. G. Phys. Rev. Lett. 1994, 73, 352

(33) Shaw, D. J. Introduction to Colloidal and Surface Science; Butterworth: Boston, MA, 1980.

(34) Agarwal, G. S.; Jha, S. S. Solid State Commun. 1982, 41, 499.

(35) Piron, A.; Brevet, P. F.; Girault, H. H. J. Electroanal. Chem. 1996, $409,123$. 